\title{
Electric and magnetic Landau-gauge gluon propagators in finite-temperature SU(2) gauge theory
}

\author{
Attilio Cucchieri ${ }^{a b}$, Tereza Mendes ${ }^{* a}$ \\ ${ }^{a}$ Instituto de Física de São Carlos, Universidade de São Paulo, \\ Caixa Postal 369, 13560-970 São Carlos, SP, Brazil \\ ${ }^{b}$ Ghent University, Department of Physics and Astronomy, \\ Krijgslaan 281-S9, 9000 Gent, Belgium \\ E-mail: attiliodifsc.usp.br, mendes@ifsc.usp.br
}

\begin{abstract}
We perform lattice simulations in pure-SU(2) Yang-Mills theory to investigate how the infrared behavior of electric and magnetic gluon propagators in Landau gauge is affected by temperature. We consider the largest lattices to date, in an attempt to keep systematic errors under control. Electric and magnetic screening masses are calculated through an Ansatz from the zero-temperature case, based on complex-conjugate poles for the momentum-space propagators. As recently reported in [1], we find good fits to the proposed form at all temperatures considered, with different ratios of real to imaginary part of the pole masses for the longitudinal (electric) and transverse (magnetic) propagators. The behavior of the magnetic propagator $D_{T}(p)$ is in agreement with the dimensional-reduction picture, showing infrared suppression (with a turnover in momentum) and violation of spectral positivity at all nonzero temperatures considered. The longitudinal propagator $D_{L}(p)$ appears to reach a plateau at small momenta and is subject to severe finite- $N_{t}$ effects around the critical temperature $T_{c}$. As a consequence, only lattices with temporal extent $N_{t}>8$ seem to be free from systematic errors. After these errors are removed, the infrared-plateau value is considerably reduced around the transition and the sharp peak observed previously for this quantity at $T_{c}$ is no longer present. The resulting infrared behavior for $D_{L}(p)$ at $T_{c}$ is essentially the same as for $0.5 T_{c}$. An investigation of the temperature range between $0.5 T_{c}$ and $T_{c}$ reveals that a less pronounced (finite) peak may occur at smaller temperatures, e.g. $T \approx 0.9 T_{c}$.
\end{abstract}

The many faces of $Q C D$

November 2-5, 2010

Gent Belgium

\footnotetext{
*Speaker.
} 


\section{Introduction}

At zero temperature, Landau-gauge gluon and ghost propagators are believed to be closely related to the confinement mechanism in Yang-Mills theories, following the so-called GribovZwanziger scenario $[2,3]$. A key feature of this scenario, formulated for momentum-space propagators, is that the gluon propagator $D(p)$ should be suppressed in the infrared limit. Such a suppression is associated with violation of spectral positivity, which is commonly regarded as an indication of gluon confinement. Lattice studies (see [4] for a review) have confirmed the suppression of $D(p)$ in the infrared limit and have also observed violation of reflection positivity for the real-space gluon propagator [5]. The infrared data for $D(p)$ are well fitted by a Gribov-Stingl form (see e.g. [6]), which generalizes the form originally proposed by Gribov, based on a propagator with a pair of complex-conjugate poles. These poles can be associated with complex values for dynamically generated masses, a behavior in agreement with the massive (or decoupling) solution of Schwinger-Dyson equations [7]. The same behavior is obtained in the refined Gribov-Zwanziger framework [8,9]. (See also [10] for a very recent proposal in maximally Abelian gauge.)

At high temperatures, on the other hand, one expects to observe Debye screening of the color charge, signaled by screening masses/lengths that can in principle be obtained from the gluon propagator [11]. More specifically, chromoelectric (resp. chromomagnetic) screening will be related to the longitudinal (resp. transverse) gluon propagator computed at momenta with null temporal component, i.e. with $p_{0}=0$ (soft modes). In particular, we expect the real-space longitudinal propagator to fall off exponentially at long distances, defining a (real) electric screening mass, which can be calculated perturbatively to leading order. Also, according to the $3 \mathrm{~d}$ adjoint-Higgs picture for dimensional reduction, we expect the transverse propagator to show a confining behavior at finite temperature, in association with a nontrivial magnetic mass (see e.g. [12]). We note that these propagators are gauge-dependent quantities, and the (perturbative) prediction that the propagator poles should be gauge-independent must be checked, by considering different gauges.

Although the nonzero- $T$ behavior described above has been verified for various gauges and established at high temperatures down to around twice the critical temperature $T_{c}[12,13]$, it is not clear how a screening mass would show up around $T_{c}$. In the following, we try to use the knowledge gained in the study of the zero-temperature case to define temperature-dependent masses for the region around and below the critical temperature. We review briefly the existing lattice results for this temperature range, present our fitting form for the infrared region, show our preliminary results for gluon propagators on large lattices for several values of the temperature and draw our conclusions. A more detailed analysis and additional data will be presented shortly elsewhere [14].

\section{Gluon propagators around and below $T_{c}$}

The behavior of Landau-gauge gluon and ghost propagators around the critical temperature $T_{C}$ has been investigated in [15]. That study showed a stronger infrared suppression for the transverse propagator $D_{T}(p)$ than for the longitudinal one $D_{L}(p)$, confirming the dimensional-reduction picture also at smaller temperatures. [We note here that a recent study [16] discusses whether this suppression is consistent with $D_{T}(0)=0$ and investigates Gribov-copy effects for the propagators.] It was also found that the ghost propagator is insensitive to the temperature. For the longitudinal 
gluon propagator, a very interesting behavior was seen: the data approach a plateau (as a function of the momentum) in the infrared region and, as a function of temperature, this plateau shows a sharp peak around the critical temperature. The exact behavior around $T_{c}$ (e.g. whether the peak turns into a divergence at infinite volume) could not be determined, since relatively small lattices were used. All studies mentioned so far are for SU(2) gauge theory. The momentum-space expressions for the transverse and longitudinal gluon propagators $D_{T}(p)$ and $D_{L}(p)$ can be found e.g. in [15].

More recently, in [17], further simulations around $T_{c}$ confirmed the above results, and lattice data for the gluon propagator were used to construct an order parameter for the chiral/deconfinement transition. More precisely, the authors use a much finer resolution around $T_{c}$ and consider the $\mathrm{SU}(2)$ and $\mathrm{SU}(3)$ cases. A check of their calculation is done for the electric screening mass, taken as $D_{L}(0)^{-1 / 2}$ and extracted from the data, where only the $p=0$ raw data point is used. The considered lattice sizes are still moderate. [We also mention a very recent study of the $S U(3)$ case, presented in [18].]

Of course, even if an exponential fit of the (real-space) longitudinal gluon propagator works at high temperature, implying that $D_{L}(0)^{-1 / 2}$ is proportional to the electric screening mass in this limit, it is not obvious that this should hold at $T \gtrsim T_{c}$. One should therefore consider more general fits. At $T=0$, the momentum-space propagator is well fitted by a Gribov-Stingl form (see e.g. [6]), allowing for complex-conjugate poles

$$
D_{L, T}(p)=C \frac{1+d p^{2 \eta}}{\left(p^{2}+a\right)^{2}+b^{2}} .
$$

This expression corresponds to two poles, at masses $m^{2}=a \pm i b$, where $m=m_{R}+i m_{I}$. The mass $m$ thus depends only on $a, b$ and not on the normalization $C$. The parameter $\eta$ should be 1 if the fitting form also describes the large-momenta region (from our infrared data we get $\eta \neq 1$ ). For consistency with the usual definition of electric screening mass, we expect to observe $m_{I} \rightarrow 0$ $(b \rightarrow 0)$ for the longitudinal gluon propagator at high temperature. Clearly, if the propagator has the above form, then the screening mass defined by $D_{L}(0)^{-1 / 2}=\sqrt{\left(a^{2}+b^{2}\right) / C}$ mixes the complex and imaginary masses $m_{R}$ and $m_{I}$ and depends on the (a priori arbitrary) normalization $C$.

\section{Results}

We have considered the pure $\mathrm{SU}(2)$ case, with a standard Wilson action. For our runs we employ a cold start, performing a projection on positive Polyakov loop configurations. Also, gauge fixing is done using stochastic overrelaxation and the gluon dressing functions are normalized to 1 at $2 \mathrm{GeV}$. We take $\beta$ values in the scaling region and lattice sizes ranging from $N_{s}=48$ to 192 and from $N_{t}=2$ to 16 lattice points, respectively along the spatial and along the temporal directions.

We note that we have improved our procedure for determining the physical temperature $T$. Instead of using the value of the lattice spacing $a$ in physical units to obtain $T=1 / N_{t} a$, we evaluate the ratio $T / T_{c}$ for a given pair $\left(\beta, N_{t}\right)$ by expressing $T$ in terms of the lattice string tension $\sigma$. More precisely, we consider the ratio $T / T_{c}=\sqrt{\sigma_{c}} / \sqrt{\sigma}$, where $\sigma$ is the (lattice) string tension evaluated at $\left(\beta, N_{t}\right)$ and $\sqrt{\sigma_{c}}$ is evaluated at the critical coupling $\beta_{c}$ for the same $N_{t}$. In this way, one avoids the inconsistency of obtaining different values for the physical critical temperature $T_{c}$ for different 

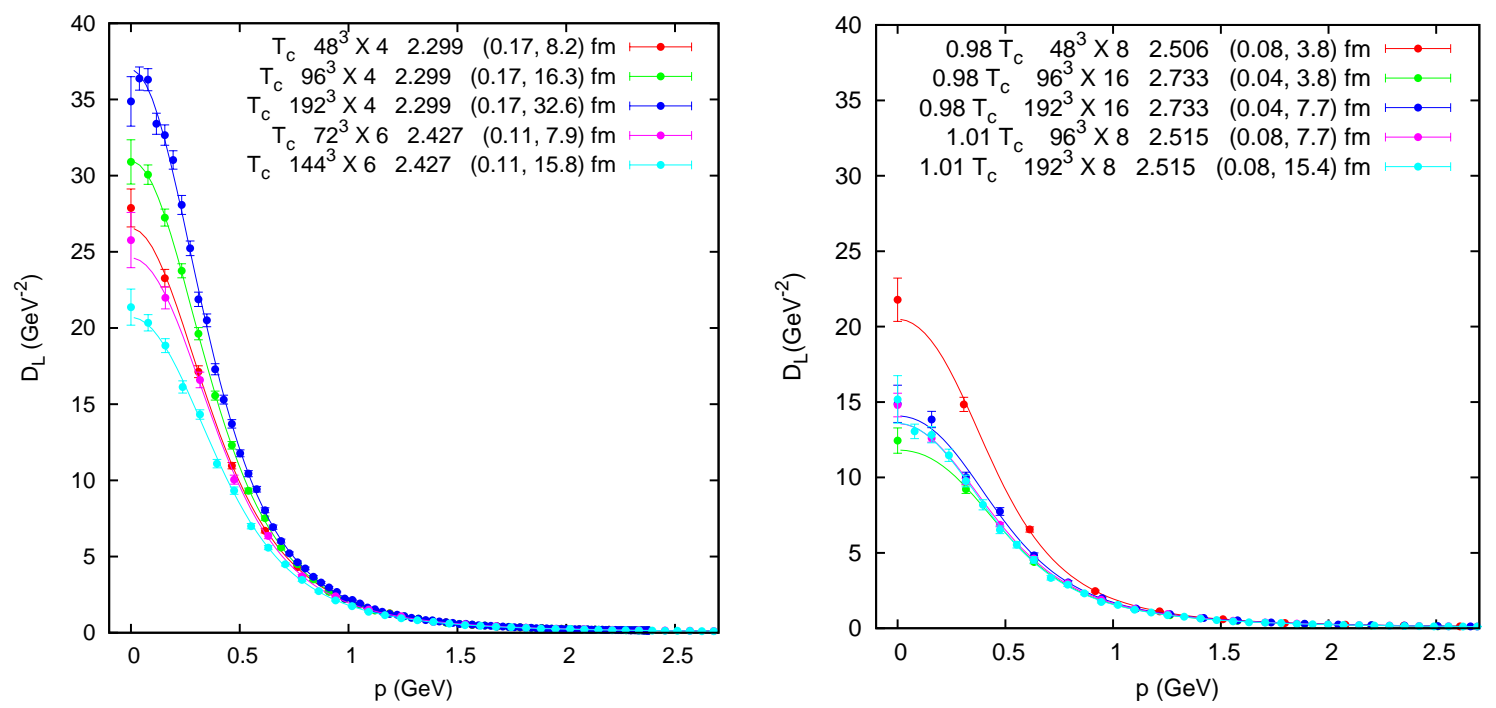

Figure 1: Longitudinal gluon propagator at and around $T_{c}$, for various lattice sizes and values of $\beta$. Values for the temperature, $N_{s}^{3} \times N_{t}, \beta$, lattice spacing $a$ and spatial lattice size $L$ (both in fm, in parentheses) are given in the plot labels. Lattices with $N_{t}<8$ and with $N_{t} \geq 8$ are shown respectively in the left and right panel.

$N_{t}$ 's. We have taken the $\beta_{c}$ values for the various $N_{t}$ 's from [19]. The string tension has been evaluated using the fit given in [20]. This leads to slightly different values of the ratio $T / T_{c}$ as compared with $[1,15]$.

All our data have been fitted to a Gribov-Stingl behavior, as described in the previous section (see Eq. 2.1). These fits are shown here in all plots of $D_{L, T}(p)$, whereas a detailed discussion of the associated masses $m_{R}, m_{I}$ will be presented elsewhere [14]. We generally find good fits to the Gribov-Stingl form (including the full range of momenta), with nonzero real and imaginary parts of the pole masses in all cases. For the transverse propagator $D_{T}(p)$, the masses $m_{R}$ and $m_{I}$ are of comparable size (around 0.6 and $0.4 \mathrm{GeV}$ respectively). The same holds for $D_{L}(p)$, but in this case the relative size of the imaginary mass seems to decrease with increasing temperature.

Our runs were initially planned under the assumption that a temporal extent $N_{t}=4$ might be sufficient to observe the infrared behavior of the propagators. (Our goal was, then, to increase $N_{s}$ significantly, to check for finite-size effects.) For this value of $N_{t}$, the chosen $\beta$ values: 2.2615 , $2.2872,2.299,2.3045,2.313,2.333,2.5058$ yield temperatures respectively of $0.92,0.96,1.00$, $1.02,1.05,1.12,1.98$ times the critical temperature $T_{c}$. (See comment above about slight differences in values of $T / T_{c}$ for a given $\beta$ in our newest analysis when compared with [1,15].) As seen in Fig. 1, the assumption that $N_{t}=4$ might be enough is not verified for the longitudinal propagator around the critical temperature, especially in the case of larger $N_{s}$. Indeed, as $N_{s}$ is doubled from 48 to 96 and then to 192, we see in Fig. 1 (left) that the infrared value of $D_{L}(p)$ changes drastically, resulting in a qualitatively different curve at $N_{s}=192$, apparently with a turnover in momentum. (Also, in this case the real-space longitudinal propagator manifestly violates reflection positivity.) We took this as an indication that our choice of $N_{t}=4$ was not valid and therefore considered larger values of $N_{t}$. Note that, due to the improved method for introducing physical units, the data 

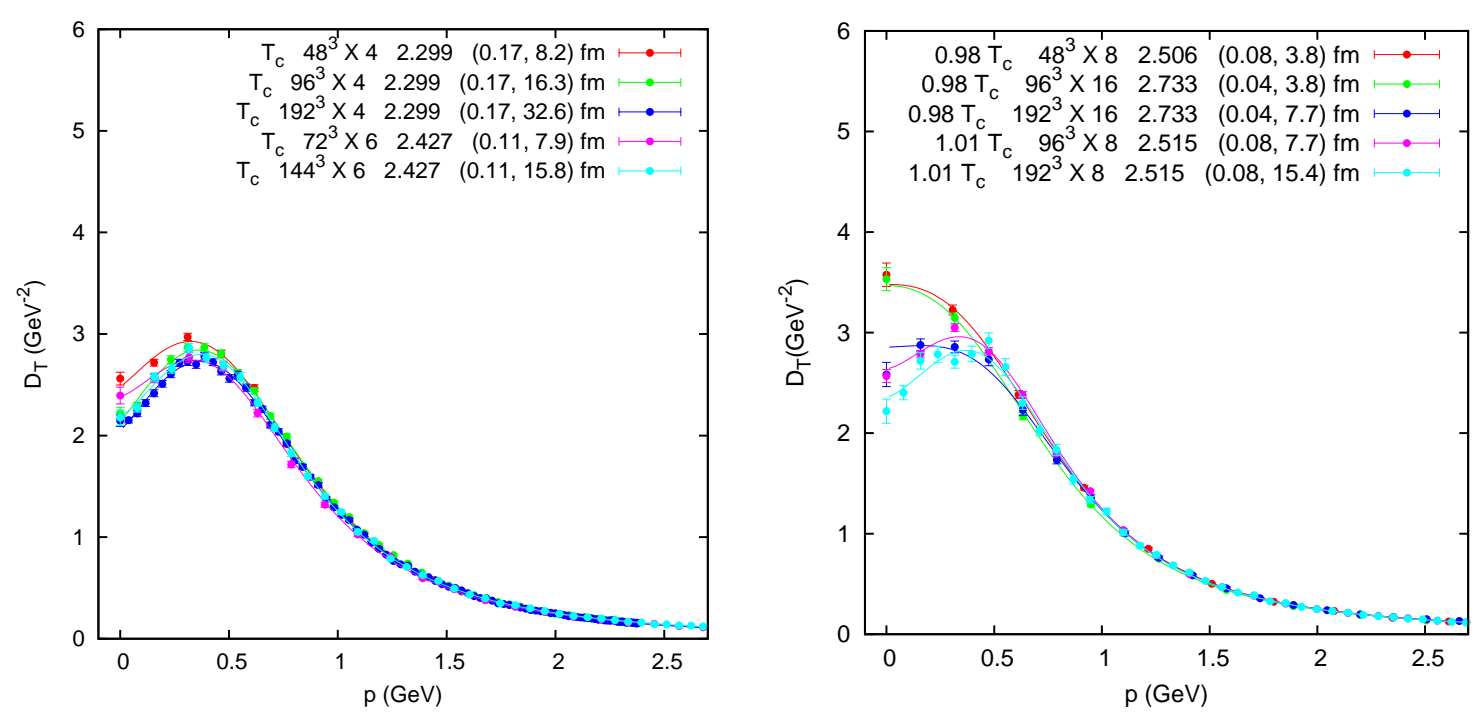

Figure 2: Transverse gluon propagator at and around $T_{c}$, for various lattice sizes and values of $\beta$. Values for the temperature, $N_{s}^{3} \times N_{t}, \beta$, lattice spacing $a$ and spatial lattice size $L$ (both in $\mathrm{fm}$, in parentheses) are given in the plot labels. Lattices with $N_{t}<8$ and with $N_{t} \geq 8$ are shown respectively in the left and right panel.

for $N_{t}<8$ (left panel of Fig. 1) lie exactly at $T_{c}$, while the data at $N_{t} \geq 8$ (right panel) are at $0.98 T_{c}$ and $1.01 T_{c}$.

As can be seen in Fig. 1 (right), we obtain in this way a different picture for the critical behavior of $D_{L}(p)$. Once we use values of $N_{t}$ that are large enough - i.e. $N_{t}>8$ for $T \lesssim T_{c}$ and $N_{t} \geq 8$ for $T \gtrsim T_{c}$ - the curve stabilizes within statistical errors for four different combinations of parameters. In particular, this includes the two curves at fixed physical volume (the blue and the magenta curves). It is interesting to note that the $N_{s}$ effects at $T_{c}$ are significant for $N_{t}=6$ (with opposite sign with respect to the $N_{t}=4$ case) and are still present for $N_{t}=8$ (and maybe also for $N_{t}=16$ ) slightly below $T_{c}$, but not immediately above $T_{c}$. Note also that the curves corresponding to the smallest physical spatial size (i.e. around $4 \mathrm{fm}$ ), may show mild finite-physical-size effects.

In Fig. 2 we show our data for the transverse propagator $D_{T}(p)$ at the critical temperature. In this case, the finite-physical-size effects are more pronounced and, in particular, the lattices with the smallest physical spatial size (the red and green curves on the right) show qualitatively different behavior when compared to the other curves. On the other hand, $D_{T}(p)$ does not seem to suffer from the same small- $N_{t}$ effects as $D_{L}(p)$. Also, we see clearly the strong infrared suppression of the propagator, with a turnover at around $400 \mathrm{MeV}$.

In summary, the transverse propagator $D_{T}(p)$ shows significant finite-physical-size effects at $T_{c}$, while the longitudinal propagator $D_{L}(p)$ is subject to two sources of systematic errors for small $N_{t}$ : "pure" small- $N_{t}$ effects (associated with discretization errors) and strong dependence on the spatial lattice size $N_{s}$ at fixed $N_{t}$, when this value of $N_{t}$ is smaller than 16. The latter effect was observed only at $T \lesssim T_{c}$, whereas the former is present in a wider region around $T_{c}$. For all investigated values of the temperature, $D_{L}(p)$ seems to reach a plateau at small $p$.

The plateau value drops significantly for $T \gtrsim T_{c}$ and then shows a steady decrease. The behavior of $D_{L}(p)$ and $D_{T}(p)$ for temperatures above $T_{c}$ is shown in Fig. 3. We see, again, that $D_{T}(p)$ 

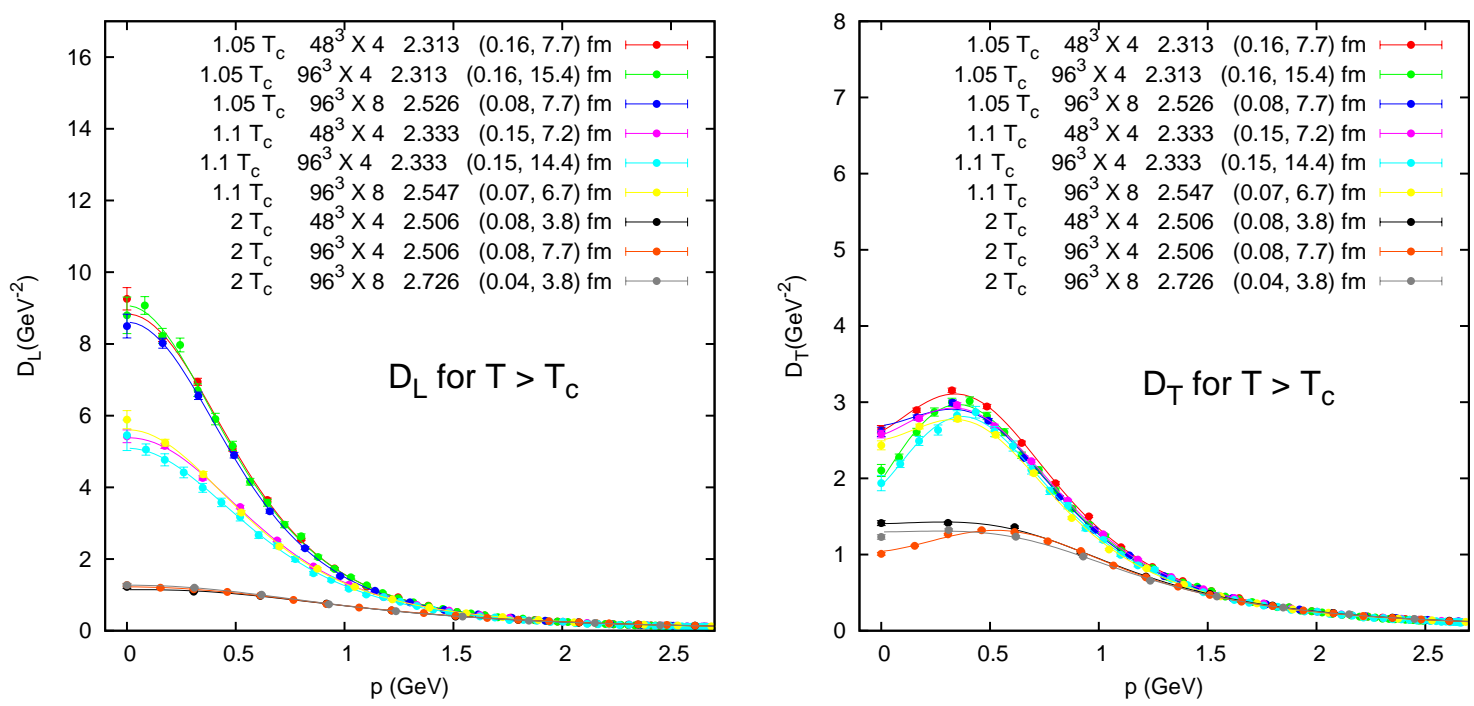

Figure 3: Longitudinal (left) and transverse (right) gluon propagator for $T>T_{c}$. Values for the temperature, $N_{s}^{3} \times N_{t}, \beta$, lattice spacing $a$ and spatial lattice size $L$ (both in fm, in parentheses) are given in the plot labels.

shows finite-physical-size effects for the smaller lattices (especially for spatial sizes below $4 \mathrm{fm}$, but also for $8 \mathrm{fm}$ when compared with $15 \mathrm{fm})$. In the $D_{L}(p)$ data, on the contrary, there are no visible systematic effects for the considered lattices at these values of the temperature.

We also did runs at low temperatures, namely at $T=0,0.25 T_{c}$ and $0.5 T_{c}$. We show combined $D_{L}(p)$ and $D_{T}(p)$ data for these runs in Fig. 4. We see that $D_{L}(p)$ increases as the temperature is switched on, while $D_{T}(p)$ decreases slightly, showing a clear turnover point at around $350 \mathrm{MeV}$. (Note that the runs at $0.5 T_{c}$ on $48^{3} \times 8$ lattices for $\beta=2.299,2.301$ are equivalent.) As pointed out before in [1], the infrared behavior of $D_{L}(p)$ remains unchanged (within errors) from $0.5 T_{c}$ to $T_{c}$. This can be seen on the bottom right plot of Fig. 4, where we show (for clarity) only lattices with large enough $N_{t}$ and the largest physical size. More precisely, we take $\beta=2.299$ on a $96^{3} \times 8$ lattice, corresponding to $0.5 T_{c}$, and $\beta=2.515$ on a $192^{3} \times 8$ lattice, corresponding to $1.01 T_{c}$. We see that, whereas the behavior of $D_{T}(p)$ is consistent with a steady monotonic decrease with temperature, the fact that $D_{L}(p)$ stays invariant might suggest a flat curve for the infrared-plateau value of the longitudinal propagator as a function of temperature below $T_{c}$.

To investigate the issue, we have performed runs at other values ot $T \leq T_{c}$. We have considered several values of $T / T_{c}$, and studied the dependence of the infrared-plateau value with $T / T_{c}$. In Fig. 5 , we show data for $D_{L}(0)$ for all our runs on the left-hand side, and for the region around $T_{c}$ on the right. We group together results from runs using the same value of $N_{t}$, and indicate them by the label "DL0_ $N_{t}$ ". The data points indicated with "sym" correspond to symmetric lattices, i.e. to the zero-temperature case. Note that results for different $N_{s}$ 's at fixed $N_{t}$ may not fall on top of each other, which gives us an indication of the systematic errors discussed above. These are especially serious for $N_{t}=4$ around $T_{c}$ (red points). We see that, surprisingly, the maximum value of $D_{L}(0)$ is not attained for $T=T_{c}$ - as might have appeared to be the case from the $N_{t}=4$ lattices only - and it does not describe a flat curve from $0.5 T_{c}$ to $T_{c}$, as could be expected by looking at the bottom right plot in Fig. 4 . Rather, it seems to lie at about $0.9 T_{c}$. Also, it clearly corresponds to a 

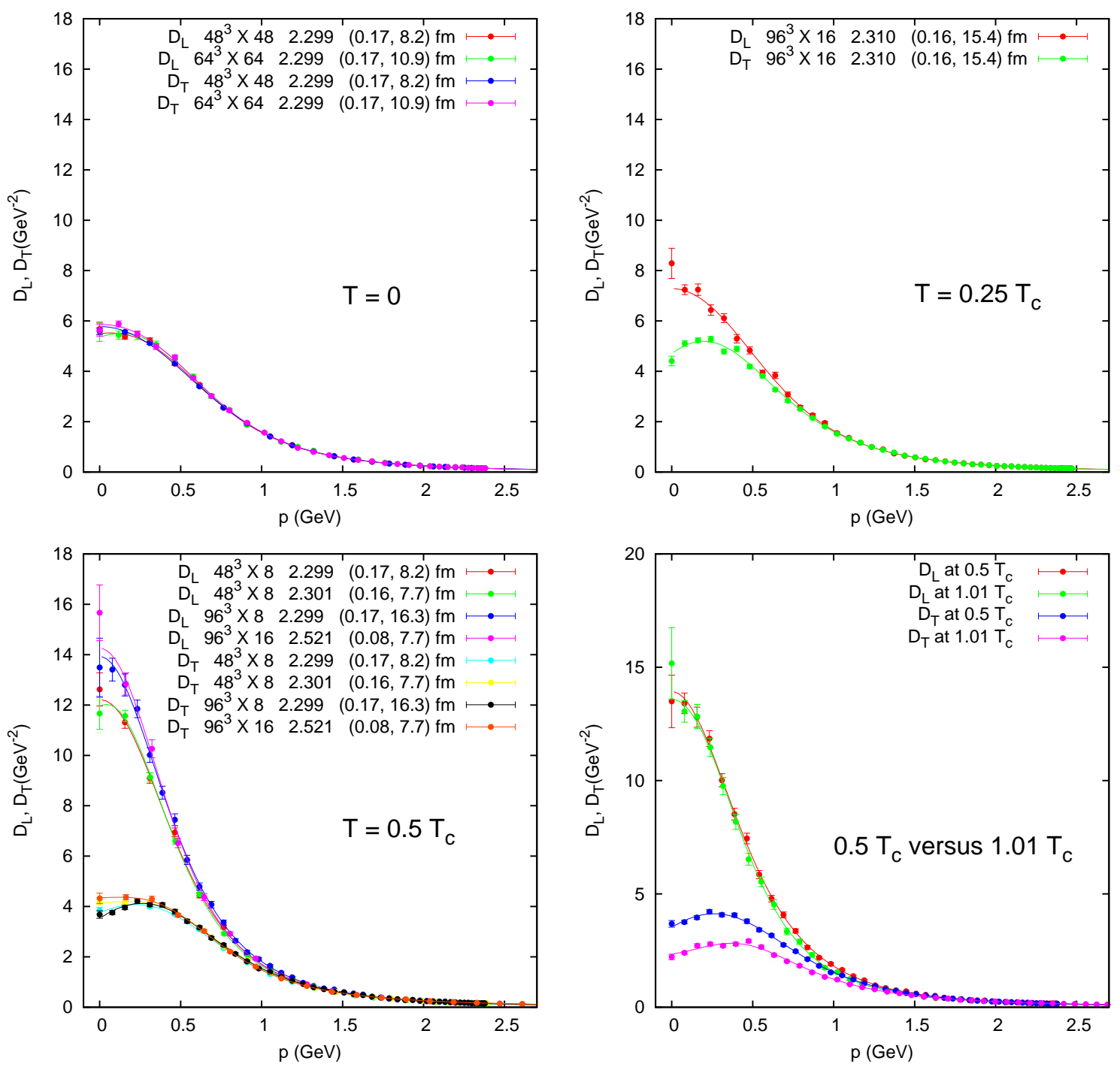

Figure 4: Longitudinal and transverse gluon propagators at $T=0$ (top left), $T=0.25 T_{c}$ (top right) and $T=0.5 T_{c}$ (bottom left). Curves for $T=0.5 T_{c}$ and $1.01 T_{c}$ are shown together for comparison on the bottom right. Values for $N_{s}^{3} \times N_{t}, \beta$, lattice spacing $a$ and spatial lattice size $L$ (both in $\mathrm{fm}$, in parentheses) are given in the plot labels, with the exception of the bottom right plot, which is described in the text.

finite peak, which does not turn into a divergence as $N_{s}$ is increased at fixed $N_{t}$.

Finally, we also looked at the real-space propagators. We find clear violation of reflection positivity for the transverse propagator at all temperatures. For the longitudinal propagator, positivity violation is observed unequivocally only at zero temperature and for a few cases around the critical region, in association with the severe systematic errors discussed above. For all other cases, there is no violation within errors. Also, we always observe an oscillatory behavior, indicative of a complex-mass pole. Typical curves for the longitudinal and transverse propagators in real space are shown (for $T=0.25 T_{c}$ ) in Fig. 6 . 

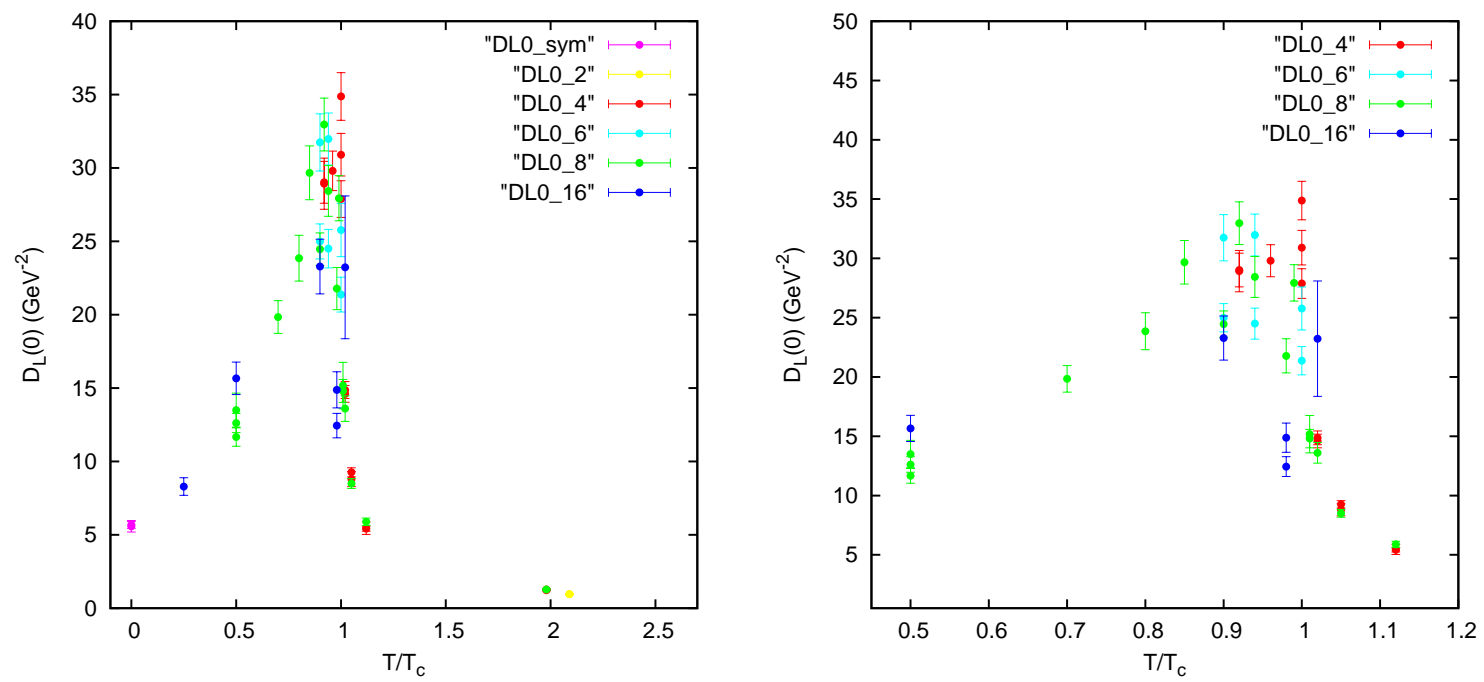

Figure 5: Infrared-plateau value for the longitudinal gluon propagator [estimated by $D_{L}(0)$ ] as a function of the temperature for the full range of $T / T_{c}$ values (left) and for the region around $T_{c}$ (right). Data points from runs at the same value of $N_{t}$ are grouped together and indicated by the label "DL0_N", where "sym" is used to indicate symmetric lattices (i.e. $T=0$ ).
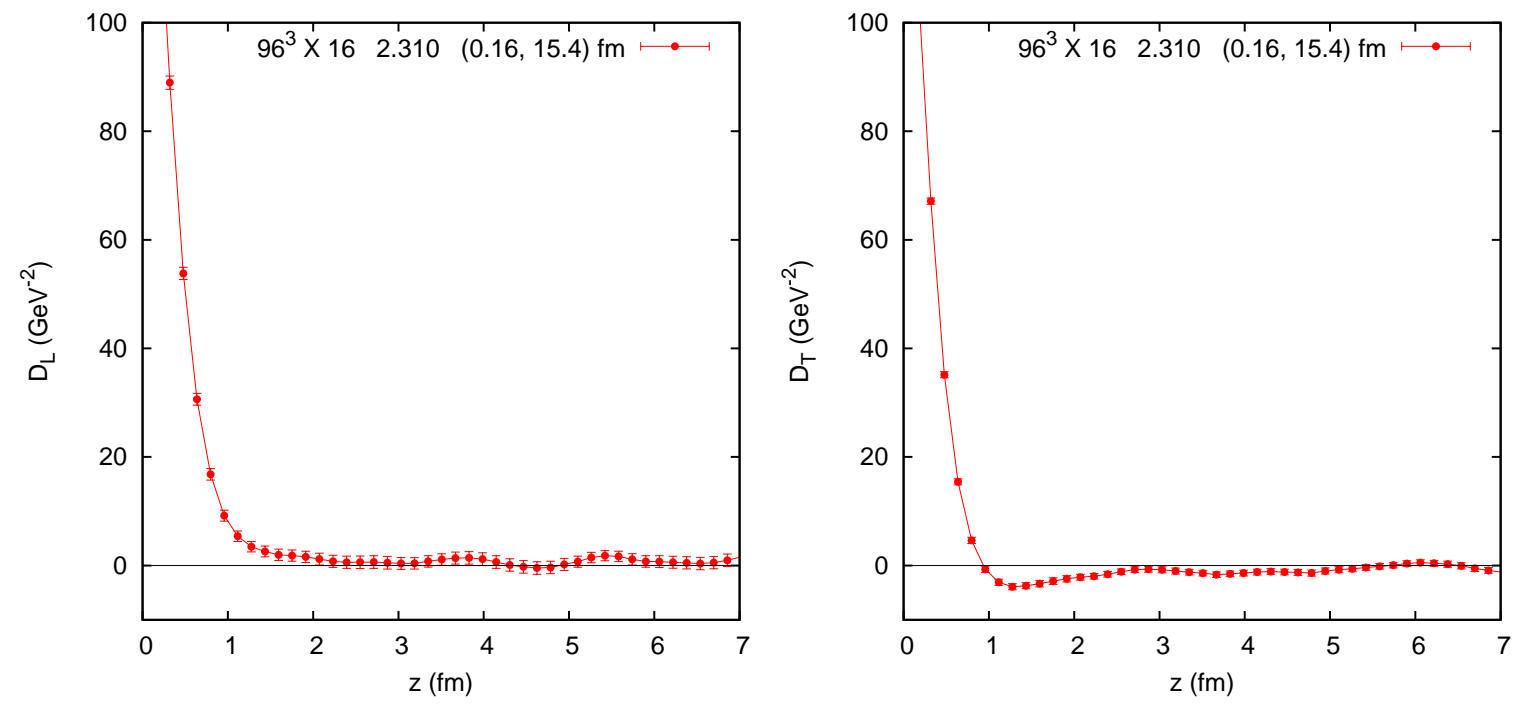

Figure 6: Longitudinal (left) and transverse (right) gluon propagator in real space for $T=0.25 T_{c}$. Values for $N_{s}^{3} \times N_{t}, \beta$, lattice spacing $a$ and spatial lattice size $L$ (both in $\mathrm{fm}$, in parentheses) are given in the plot labels. Note that the solid lines are not fits. 


\section{Conclusions}

The transverse gluon propagator $D_{T}(p)$ shows infrared suppression and a turnover in momentum (in agreement with the dimensional-reduction picture) at all nonzero temperatures considered. Also, it exhibits violation of reflection positivity as a function of real-space coordinates in all cases studied. The longitudinal propagator $D_{L}(p)$, on the contrary, appears to reach a plateau at small momenta, and does not in general show violation of reflection positivity. We have obtained good fits of our data to a Gribov-Stingl form, with comparable real and imaginary parts of the pole masses, also in the longitudinal-propagator case. This is in contrast with an electric screening mass defined by the expression $D_{L}(0)^{-1 / 2}$, which moreover may contain significant finite-size effects.

The data for $D_{L}(p)$ are subject to sizeable discretization errors around the critical temperature. In particular, a severe dependence on the aspect ratio $N_{t} / N_{s}$ is seen at $T \lesssim T_{c}$ for the smaller fixed values of $N_{t}$. As a result, only lattices with $N_{t}>8$ seem to be free from systematic errors. After these errors are removed, we see an infrared value about $50 \%$ smaller than before. As noted in [1], this might suggest that there is no jump in the infrared value of $D_{L}(p)$ as $T \rightarrow T_{c}$ from below, since the resulting infrared behavior at $T_{c}$ is essentially the same as at $0.5 T_{c}$ (see Fig. 4). [We note that all previous studies of $D_{L}(p)$ around $T_{c}$ had employed $N_{t} \leq 4$.] An investigation of the temperature range between $0.5 T_{c}$ and $T_{c}$ shows, nevertheless, a (finite) maximum of the plateau value $D_{L}(0)$ at around $0.9 T_{c}$ (see Fig. 5).

\section{Acknowledgements}

The authors thank agencies FAPESP and CNPq for financial support and the organizers of “The many faces of QCD” for a pleasant and stimulating meeting. Our simulations were performed on the new CPU/GPU cluster at IFSC-USP (obtained through a FAPESP grant).

\section{References}

[1] A. Cucchieri, T. Mendes, PoS LATTICE2010, 280 (2010).

[2] D. Zwanziger, Nucl. Phys. B 364, 127 (1991).

[3] D. Zwanziger, arXiv:1103.1137 [hep-th].

[4] A. Cucchieri, T. Mendes, PoS QCD-TNT09, 026 (2009).

[5] K. Langfeld, H. Reinhardt, J. Gattnar, Nucl. Phys. B 621, 131 (2002); A. Cucchieri, T. Mendes, A. R. Taurines, Phys. Rev. D 71, 051902 (2005); P. J. Silva, O. Oliveira, PoS LAT2006, 075 (2006); A. Sternbeck et al., PoS LAT2006, 076 (2006); P. O. Bowman et al., Phys. Rev. D 76, 094505 (2007).

[6] A. Cucchieri, T. Mendes, A. R. Taurines, Phys. Rev. D 67, 091502 (2003).

[7] A. C. Aguilar, D. Binosi, J. Papavassiliou, Phys. Rev. D 78, 025010 (2008).

[8] D. Dudal, J. A. Gracey, S. P. Sorella, N. Vandersickel, H. Verschelde, Phys. Rev. D78, 065047 (2008).

[9] D. Dudal, J. A. Gracey, S. P. Sorella, N. Vandersickel, H. Verschelde, Phys. Rev. D78, 125012 (2008).

[10] K. -I. Kondo, arXiv:1103.3829 [hep-th].

[11] D. J. Gross, R. D. Pisarski, L. G. Yaffe, Rev. Mod. Phys. 53, 43 (1981). 
[12] A. Cucchieri, F. Karsch, P. Petreczky, Phys. Rev. D 64, 036001 (2001).

[13] U. M. Heller, F. Karsch, J. Rank, Phys. Rev. D 57, 1438 (1998); A. Cucchieri, F. Karsch, P. Petreczky, Phys. Lett. B 497, 80 (2001).

[14] A. Cucchieri, T. Mendes, to be submitted.

[15] A. Cucchieri, A. Maas, T. Mendes, Phys. Rev. D 75, 076003 (2007).

[16] V. G. Bornyakov, V. K. Mitrjushkin, arXiv:1011.4790 [hep-lat].

[17] C. S. Fischer, A. Maas, J. A. Muller, Eur. Phys. J. C 68, 165 (2010).

[18] V. G. Bornyakov, V. K. Mitrjushkin, arXiv:1103.0442 [hep-lat].

[19] J. Fingberg, U. M. Heller, F. Karsch, Nucl. Phys. B392, 493-517 (1993).

[20] J. C. R. Bloch, A. Cucchieri, K. Langfeld, T. Mendes, Nucl. Phys. B687, 76-100 (2004).

[21] A. Cucchieri, T. Mendes, Phys. Rev. Lett. 100, 241601 (2008). 